\title{
ON THE DIELECTRIC CONSTANT OF LIQUID AMMONIA
}

\author{
J.S. PERKYNS, P.G. KUSALIK and G.N. PATEY \\ Department of Chemistry, University of British Columbia, Vancouver, British Columbia, Canada V6T IY6
}

Received 3 June 1986

\begin{abstract}
The purpose of this paper is to examine a simple polar-polarizable model for liquid ammonia. Theoretical values of the mean molecular dipole moment and the static dielectric constant are reported for temperatures ranging from $-70^{\circ} \mathrm{C}$ to $35^{\circ} \mathrm{C}$. The theoretical results are obtained by solving the self-consistent mean field (SCMF) equations within the reference hypernetted-chain (RHNC) approximation. It is shown that molecular polarizability has a large influence upon the properties of this system. The theoretical dielectric constants are in fair agreement with experiment over the temperature range considered.
\end{abstract}

\section{Introduction}

In several recent articles $[1-3]$ it has been shown that theoretical estimates for the dielectric constant, $\epsilon$, of dense multipolar polarizable fluids can be obtained using a self-consistent mean field (SCMF) approximation coupled with integral equation techniques. Other physically interesting quantities such as the mean molecular dipole moment in the liquid state are also given by this theoretical approach. Essentially, the SCMF approximation reduces the many-body energy of the polarizable system to a sum over an effective pairwise additive potential. The integral equation methods are used to calculate the properties of the fluid characterized by this effective pair interaction. For polarizable dipolar-quadrupolar fluids, it has been found [2] that the dielectric constants given by this approach are in good agreement with "exact" computer simulation results, provided that the integral equation theory employed gives an accurate description of the effective system. In fact, it has been demonstrated [3] that the inaccuracies in this method arise almost totally from the integral equation approximations and not from the SCMF reduction of the many-body potential.

It has also been found that a polarizable dipolarquadrupolar hard-sphere model gives dielectric constants in surprisingly good agreement with experimental results for liquid water over a wide range of temperature and density $[1,4]$. Furthermore, it is clear that molecular polarizability makes an important contribution to the static dielectric constant. The average dipole moment in the liquid state is considerably larger than the gas-phase value [1], and both approximate theories $[1,4]$ and computer simulations [5] have shown that models for water which ignore polarizability seriously underestimate the dielectric constant.

In view of the importance of these observations in. the dielectric theory of liquid water, it is of interest to see if similar effects are found for other substances. Therefore, the purpose of this paper is to examine the properties of a similar polarizable dipolar-quadrupolar hard-sphere model for liquid ammonia, and to compare with experimental results over a significant temperature rańge. The mean dipole moments and dielectric constants for this model are obtained using SCMF theory together with the reference hypernetted-chain (RHNC) approximation. The RHNC approximation has recently been solved for non-polarizable dipolar [6] and dipolar-quadrupolar [7] fluids and the dielectric constants obtained are generally in good agreement with computer simulation results. We note that for systems characterized by large dipole but relatively small quadrupole moments the full RHNC approximation greatly improves upon the linearized and quadratic versions of the theory (i.e. RLHNC and RQHNC, respectively) [8] used in earlier work [1-3]. Thus we would expect the SCMF/RHNC calcualtions to give accurate resolutions for the present model. 


\section{The model and theory}

We consider an ammonia molecule to be a hard sphere of diameter $d$, with a permanent dipole moment, $\boldsymbol{\mu}$, a quadrupole moment, $Q$, and a point polarizability, $\boldsymbol{\alpha}$, embedded at the center. Ammonia has $\mathrm{C}_{3 \mathrm{v}}$ symmetry and this allows the quadrupolar interactions to be expressed in terms of a single scalar, $Q$ [9]. The polarizability tensor $\boldsymbol{\alpha}$ is completely specified by $\alpha_{\|}$ and $\alpha_{\perp}$ which are, respectively, the components parallel and perpendicular to the symmetry axis. The values of the parameters used in the present calculations are summarized in table 1 . The hard-sphere diameter is somewhat arbitrary, but the value $3.2 \AA$ is a reasonable estimate based upon the radial distribution function for liquid ammonia as determined by neutron scattering experiments [10]. For all other parameters the experimental gas-phase values were used. It should be noted that the quadrupole moment for ammonia, as originally reported [15] (i.e. $Q=-2.32 \mathrm{~B}$ ), is in error and that the correct value (i.e. $Q=-3.3 \mathrm{~B}$ ) is given in ref. [12]. We emphasize this point because the erroneous value has been repeated in literature tabulations [16] and has been used in previous work [17-19] on liquid ammonia.

As mentioned above, the exact instantaneous configurational energy for fluids consisting of polarizable molecules depends upon many-body interactions [1$3,20]$. SCMF theory reduces this problem by relating the properties of the polarizable fluid to those of an effective system. For the present model, this effective system is characterized by the pair potential

Table 1

Molecular parameters for ammonia. The values given for $\mu$, $Q, \alpha_{\|}$and $\alpha_{\perp}$ are experimental gas-phase results. The hardsphere diameter, $d$, was estimated from neutron scattering data [10]

\begin{tabular}{ll}
\hline Parameter & Value \\
\hline$d$ & $3.2 \AA$ \\
$\mu$ & $1.47 \mathrm{D}[11]$ \\
$Q$ & $-3.3 \mathrm{~B}$ a) \\
$\alpha_{\|}$ & $2.412 \AA^{3}[14]$ \\
$\alpha_{\perp}$ & $2.124 \AA^{3}[14]$ \\
\hline
\end{tabular}

a) Experimental values of $-3.3 \pm 0.4 \mathrm{~B}[12]$ and $-3.25 \pm$ $0.05[13]$ have been reported in the literature.

$$
\begin{aligned}
& u_{\mathrm{e}}(12)=u_{\mathrm{HS}}(r)-\left(m_{\mathrm{e}}^{2} / r^{3}\right) \Phi^{112}(12) \\
& \quad+\left(m_{\mathrm{e}} Q / 2 r^{4}\right)\left[\Phi^{123}(12)-\Phi^{213}(12)\right] \\
& +\left(Q^{2} / 4 r^{5}\right) \Phi^{224}(12),
\end{aligned}
$$

where $u_{\mathrm{HS}}(r)$ is the usual hard-sphere interaction and the angle-dependent functions, $\Phi^{m n l}(12)$, are as defined in ref. [7]. The effective dipole moment, $m_{\mathbf{e}}$, is defined [1] such that $m_{\mathrm{e}}^{2}=\left\langle m^{2}\right\rangle$ where $m$ is the total (i.e. permanent plus induced) dipole moment of a single particle. The value of $m_{\mathrm{e}}$ consistent with a given polarizability, $\boldsymbol{\alpha}$, is determined by the exact relationships [1]

$\boldsymbol{m}^{\prime}=\boldsymbol{\mu}+C\left(m^{\prime}\right) \boldsymbol{\mu} \cdot \boldsymbol{\alpha}^{\prime}$,

$\boldsymbol{\alpha}^{\prime}=\boldsymbol{\alpha}+C\left(m^{\prime}\right) \boldsymbol{\alpha} \cdot \boldsymbol{\alpha}^{\prime}$,

$m_{\mathrm{e}}^{2}=\left(m^{\prime}\right)^{2}+3 \alpha^{\prime} k T$,

where $\boldsymbol{m}^{\prime}=\langle\boldsymbol{m}\rangle$ is the average molecular dipole moment, $\boldsymbol{\alpha}^{\prime}$ is a renormalized polarizability, $\alpha^{\prime}=\frac{1}{3} \operatorname{Tr} \boldsymbol{\alpha}^{\prime}$, and $C\left(m^{\prime}\right)$ is a scalar related to the average dipolar and quadrupolar fields experienced by a molecule. In the SCMF approximation $C\left(m^{\prime}\right)$ is given by [1]

$C\left(m^{\prime}\right)=-2\left\langle U_{\mathrm{DD}}\right\rangle_{\mathrm{e}} / m_{\mathrm{e}}^{2} N-\left\langle U_{\mathrm{DQ}}\right\rangle_{\mathrm{e}} / N m_{\mathrm{e}} m^{\prime}$,

where $N$ is the number of particles and $\left\langle U_{\mathrm{DD}}\right\rangle_{\mathrm{e}}$ and $\left\langle U_{\mathrm{DQ}}\right\rangle_{\mathrm{e}}$ are the average dipole-dipole and dipolequadrupole energies of the effective system.

In the present calculations, $\left\langle U_{\mathrm{DD}}\right\rangle_{\mathrm{e}}$ and $\left\langle U_{\mathrm{DQ}}\right\rangle_{\mathrm{e}}$ as well as other properties of the effective system are obtained by solving the RHNC approximation for the pair potential defined by eq. (1). Hence, the SCMF/ RHNC solution for the polarizable fluid has been found when eqs. (1), (2) and (3) together with the equations comprising the RHNC theory [7] are satisfied simultaneously. In practice this is carried out numerically using the procedures described in previous papers $[1,6,7]$.

It has recently been shown [3] that within the SCMF approximation the dielectric constant of the effective system can be identified as that of the polarizable fluid of interest. This result is independent of the theory used to obtain the properties of the effective system. Hence, in the present SCMF/RHNC calculations $\epsilon$ is given by the formula [8]

$(\epsilon-1)(2 \epsilon+1) / 9 \epsilon=y_{\mathrm{e}} g_{\mathrm{e}}$, 
where $y_{\mathrm{e}}=4 \pi \rho m_{\mathrm{e}}^{2} / 9 k T, \rho$ is the number density, and the Kirkwood $g$-factor for the effective system, $g_{\mathrm{e}}$, is obtained from the RHNC approximation [7].

\section{Results and discussion}

Calculations have been carried out at four points lying on the equilibrium vapour pressure curve for ammonia. The temperatures considered are $-70,-35$, 5 , and $35^{\circ} \mathrm{C}$, for which the corresponding liquid densities are [21] $0.7250,0.6840,0.6315$, and $0.5875 \mathrm{~g}$ $\mathrm{cm}^{-3}$, respectively.

The dielectric constants for the present model given by the SCMF/RHNC theory are compared with experimental values $[22,23]$ in fig. 1 . Theoretical results for the non-polarizable case (i.e., $\boldsymbol{\alpha}=0$ ) are also included in fig. 1 . It is apparent that when polarizability is ignored the theoretical dielectric constants are much smaller than the experimental values. This is consistent with earlier observations for water [1]. The dielectric constants obtained when polarizability is included in

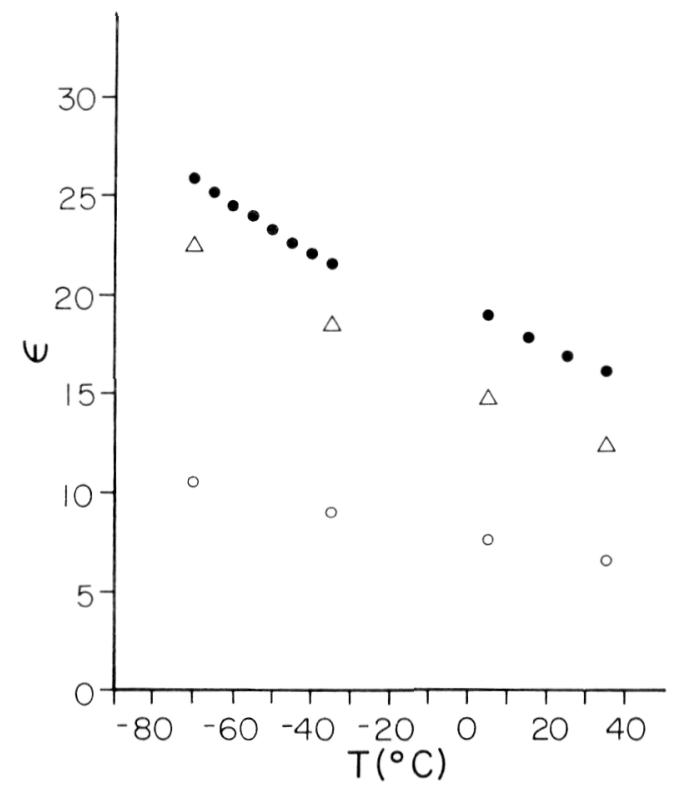

Fig. 1. The dielectric constant as a function of temperature. The open circles are SCMF/RHNC results for the non-polarizable (i.e., $\boldsymbol{\alpha}=0$ ) case. The triangles are the SCMF/RHNC results for the polarizable model. The solid dots are experimental values from the literature: points below $0^{\circ} \mathrm{C}$ are from ref. [22], points above $0^{\circ} \mathrm{C}$ from ref. [23]. the model are in much better agreement with the experimental results, but still lie under the experimental curve for all temperatures considered. The reason for this discrepancy is not clear, but it should be borne in mind that for the present model the theoretical values for $\epsilon$ are rather sensitive to the quadrupole moment rapidly increasing as $Q$ is decreased [19]. Therefore, it is possible that uncertainties in the experimental val. ue of $Q$ can account for much of the difference between the theoretical and experimental results. The experimental error bars reported in ref. [12] (see table 1) are certainly large enough for this to be true. The error bars quoted in ref. [13] are much smaller but, as pointed out by previous authors [24], these error bars seem rather small compared with the inaccuracy in quadrupole measurements for other molecules (cf. refs. $[16,25])$. Also, we note that the most recent quantum calculations [24] give $Q=-3.08 \mathrm{~B}$ which ! is somewhat smaller than the value used in the present calculations. Finally, it is worth remarking that in all likelihood the discrepancy does not arise from the RHNC approximation, since if anything this approximation tends to overestimate the dielectric constant $[6,7]$.

Several additional calculations were carried out using the erroneous value $Q=-2.32 \mathrm{~B}$ mentioned above. With this smaller quadrupole moment $\epsilon$ in-

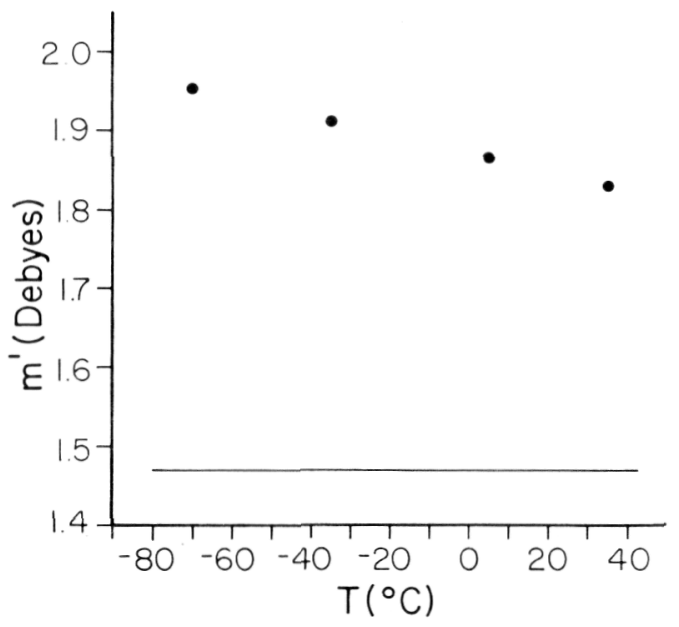

Fig. 2. The temperature dependence of the mean molecular dipole moment $m^{\prime}$. The solid dots are SCMF/RHNC results for the polarizable ammonia model. The horizontal line indicates the gas-phase value, $\mu=1.47 \mathrm{D}$. 
creases much more rapidly with decreasing temperature and is roughly twice the experimental value at $-70^{\circ} \mathrm{C}$. This demonstrates the sensitivity of $\in$ to $Q$ and serves to emphasize the importance of including the correct quadrupole moment in molecular models for ammonia..We also note that the full RHNC approximation is necessary in the present calculations. The RLHNC approximation is not sufficiently accurate for ammonia-like parameters and seriously overestimates the dielectric constant at lower temperatures.

The mean dipole moment $m^{\prime}$ is plotted as a function of temperature in fig. 2. It can be seen that $m^{\prime}$ is considerably larger than the gas-phase value (i.e. 1.47 D) but does not show a strong temperature dependence, varying only from 1.83 to $1.95 \mathrm{D}$ as the temperature drops from $35^{\circ} \mathrm{C}$ to $-70^{\circ} \mathrm{C}$. This is roughly similar to the variation observed [1] for liquid water over the $20^{\circ} \mathrm{C}$ to $125^{\circ} \mathrm{C}$ temperature range.

In summary, the present calculations have shown that in liquid ammonia, as in liquid water, molecular polarizability makes an important contribution to the static dielectric constant. Furthermore, considering that no freely adjustable parameters are included, the present model and theory gives a reasonably accurate account of the temperature dependence of $\epsilon$.

\section{Acknowledgement}

We are grateful for the financial support of the Natural Sciences and Engineering Research Council of Canada.

\section{References}

[1] S.L. Carnie and G.N. Patey, Mol. Phys. 47 (1982) 1129.
[2] J.M. Caillol, D. Levesque, J.J. Weis, P.G. Kusalik and G.N. Patey, Mol. Phys. 55 (1985) 65.

[3] G.N. Patey, D. Levesque and J.J. Weis, Mol. Phys. 57 (1986) 337.

[4] P.G. Kusalik and G.N. Patey, to be published.

[5] N. Neumann, J. Chem. Phys. 82 (1985) 5663.

[6] P.H. Fries and G.N. Patey, J. Chem. Phys. 82 (1985) 429.

[7] J.S. Perkyns, P.H. Fries and G.N. Patey, Mol. Phys. 57 (1986) 529.

[8] G. Stell, G.N. Patey and J.S. Hфye, Advan. Chem. Phys. 48 (1981) 183.

[9] A.D. Buckingham, Advan. Chem. Phys. 12 (1967) 107.

[10] A.H. Narten, J. Chem. Phys. 66 (1977) 3117.

[11] A.L. MoClellan, Tables of experimental dipole moments (Freeman, San Francisco, 1963) p. 27.

[12] S.G. Kukolich, Chem. Phys. Letters 12 (1971) 216.

[13] J.G.C.M. van Duijneveldt-van de Rijdt and F.B. van Duijneveldt, J. Mol. Struct. THEOCHEM 89 (1982) 185 , and references therein.

[14] N.J. Bridge and A.D. Buckingham, Proc. Roy. Soc. A205 (1966) 135.

[15] S.G. Kukolich, Chem. Phys. Letters 5 (1970) 401.

[16] W.H. Flygare, Chem. Rev. 74 (1974) 653.

[17] M.L. Klein, I.R. McDonald and R. Righini, J. Chem. Phys. 71 (1973) 3673.

[18] A. Hinchliffe, D.G. Bounds, M.L. Klein, I.R. McDonald and R. Righini, J. Chem. Phys. 74 (1981) 1211.

[19] G.N. Patey, D. Levesque and J.J. Weis, Mol. Phys. 38 (1979) 1635 .

[20] C.J.F. Böttcher, Theory of electric polarization, Vol. 1, 2nd Ed. (Elsevier, Amsterdam, 1973) ch. 6.

[21] C.A. Vancini, Synthesis of ammonia (MacMillan, New York, 1971) p. 169,

[22] J. Baldwin and J.B. Gill, Phys. Chem. Liquids 2 (1970) 25.

[23] R.C. Weast, ed., CRC handbook of chemistry and physics, 60th Ed. (CRC Press, Boca Raton, 1975) p. E55.

[24] G.H.F. Diercksen and A.J. Sadlej, Mol. Phys. 57 (1986) 509.

[25] A.D. Buckingham, C. Graham and J.H. Williams, Mol. Phys. 49 (1983) 703. 